\title{
The reaction of 2-naphthyl with 1,3-butadiene: a theoretical study
}

\author{
Oleinikov Artem ${ }^{1}$, Mebel Alexander ${ }^{1,2 *}$, and Azyazov Valeriy ${ }^{1,3}$ \\ ${ }^{1}$ Samara National Research University, 443086, Samara, Russian Federation \\ ${ }^{2}$ Florida International University, FL 33199, Miami, USA \\ ${ }^{3}$ Lebedev Physical Institute, 443011, Samara, Russian Federation
}

\begin{abstract}
This work is devoted to a theoretical study of the 2-naphthyl + 1,3-butadiene reaction aimed at understanding the hydrogen-loss mechanism in the reactions of $n$-naphthyl $(n=1,2)$ with 1,3-butadiene. All intermediates and transition states, their vibrational frequencies and zeropoint vibrational energies, and the potential energy diagram were calculated at the G3(MP2,CC)//B3LYP/6-311G** theoretical level. The estimate of the reaction rate constant gives the order of $10^{-13} \mathrm{~cm}^{3}$ molecule${ }^{1} \mathrm{~s}^{-1}$ at temperatures below $500 \mathrm{~K}$.
\end{abstract}

\section{Introduction}

A great amount of theoretical and experimental research in the field of polycyclic aromatic hydrocarbons (PAH) growth and soot formation have been reported in last decades. These investigations demonstrated harmful effects of PAH on the environment and human health [1-3]. Due to high thermal stability of aromatic radicals and resonantly stabilized free radicals the following mechanisms are considered important in the PAH and soot precursor formation: hydrogen abstraction-acetylene addition (HACA) [4-6], phenyl addition-cyclization [7, 8], and ethynyl addition [9]. However, more complex PAH such as three-ring phenanthrene and anthracene could not be formed through HACA-based mechanisms. In this article, the addition of a 1,3-butadiene molecule is proposed as a key step in the formation of a third aromatic ring.

Recently obtained experimental and theoretical results on the reaction of 1-naphthyl with 1,3-butadiene defy the thesis that PAH growth is predominantly a high temperature phenomenon [10] and a further support for this conclusion is provided here for the analogous reaction of 2-naphthyl.

\section{Methods}

Optimization of geometry of reactants, products, intermediates, and transition states, and zero-point vibrational energy calculations were performed by using DFT calculations at the B3LYP/6-311G** level. Single point energies were refined by using higher level of theory methods and calculated using the following scheme: $\mathrm{E}_{0}[\mathrm{G} 3(\mathrm{MP} 2, \mathrm{CC})]=\mathrm{E}$

\footnotetext{
*Corresponding author: mebela@fiu.edu
} 
$\left[\mathrm{CCSD}(\mathrm{T}) / 6-311 \mathrm{G}^{* *}\right]+\Delta \mathrm{EMP} 2+\mathrm{E}(\mathrm{ZPE})$, where $\Delta \mathrm{EMP} 2=\mathrm{E}[\mathrm{MP} 2 / \mathrm{G} 3$ Large $]-$ $\mathrm{E}\left[\mathrm{MP} 2 / 6-311 \mathrm{G}^{* *}\right]$ is a basis set correction and $\mathrm{E}(\mathrm{ZPE})$ is the zero-point energy. The calculations were performed by using the GAUSSIAN 09 [2] and MOLPRO 2010 packages [3].

Reaction rate constants for all reaction steps were computed by solving one-dimensional master equation using Rice-Ramsperger-Kassel-Marcus theory. The harmonic approximation was applied to calculate partition functions using B3LYP/6-311G** frequencies.

\section{Results}

Quantum chemical calculations at the theory level described above provide information about five hydrogen loss channels in the bimolecular reaction of 2-naphthyl with 1,3butadiene. All the investigated species and intermediates are represented in Figure 1 with relative energies given in $\mathrm{kcal} \mathrm{mol}^{-1}$ with respect to the reactants. The first channel leading to $\mathrm{P} 4+\mathrm{H}$ products starts with a small barrier (TS0-4) $3.87 \mathrm{kcal} \mathrm{mol}^{-1}$ above the reactants. This is a formation of a bond between the radical site in 2-naphthyl and a second $\mathrm{C}$ atom in 1,3-butadiene leading to intermediate W4 located in a potential well $32.78 \mathrm{kcal} \mathrm{mol}^{-1}$ below the reagents. The further development of this channel passes through a hydrogen loss barrier TS4-P4 from the attacked carbon in 1,3-butadiene. The barrier height is $32.59 \mathrm{kcal}$ $\mathrm{mol}^{-1}$ and the channel forms the $\mathrm{P} 4$ species that is $7.36 \mathrm{kcal} \mathrm{mol}^{-1}$ lower in energy than the separated reagents. The initial reactants also have a second channel leading to the P1, P2, P3 and P6 products presented in Figure 1. This channel starts with a van der Waals complex W0 located at $-1.72 \mathrm{kcal} \mathrm{mol}^{-1}$ that represents a barrier-free entry into the reaction channel. After that a bond is formed between the radical site in 2-naphthyl and the terminal carbon atom of 1,3-butadiene via the transition state TS0-1 (-1.07 kcal mol$\left.{ }^{-1}\right)$ descending into a potential well W1 at $-46.82 \mathrm{kcal} \mathrm{mol}^{-1}$. Hydrogen loss from $\mathrm{C} 1$ in 1,3-butadiene represents the reaction channel from $\mathrm{W} 1$ to the $\mathrm{P} 1+\mathrm{H}$ products (at $-8.98 \mathrm{kcal} \mathrm{mol}^{-1}$ ) via the transition state TS1-P1 located at $-5.91 \mathrm{kcal} \mathrm{mol}^{-1}$. The reaction can also continue from W1 (-46.82 $\left.\mathrm{kcal} \mathrm{mol}^{-1}\right)$ to $\mathrm{W} 2\left(-46.26 \mathrm{kcal} \mathrm{mol}^{-1}\right)$ intermediates that are close in energy and the linking transition state TS1-2 at $-32.67 \mathrm{kcal} \mathrm{mol}^{-1}$ is a trans-cis rearrangement in the 1,3-butadiene side chain. The next endoergic hydrogen loss from $\mathrm{C} 1$-carbon in butadiene pathway leads to the $\mathrm{P} 2+\mathrm{H}$ products at $-6.04 \mathrm{kcal} \mathrm{mol}^{-1}$ via transition state TS2-P2 at $-2.95 \mathrm{kcal} \mathrm{mol}^{-1}$. Other two pathways lead to P3 (1,4-dihydrophenanthrene) and P6 (1,4-dihydroanthracene). Both mechanisms involve ring closure. The channel to P3 passes via TS2-3 (-27.29 kcal $\left.\mathrm{mol}^{-1}\right)$ to $\mathrm{W} 3\left(-52.23 \mathrm{kcal} \mathrm{mol}^{-1}\right)$ with ring closure on the $\mathrm{C} 1$-carbon of the former 2naphthyl reactant and after that proceeds via hydrogen loss from the $\mathrm{C} 1$-carbon via transition state TS3-P3 $\left(-25.26 \mathrm{kcal} \mathrm{mol}^{-1}\right)$ and gives the product $\mathrm{P} 3+\mathrm{H}$ at $-25.05 \mathrm{kcal} \mathrm{mol}^{-}$ 1. The last reaction channel has the similar mechanism but the ring closure in TS2-6 (-26.24 kcal $\mathrm{mol}^{-1}$ ) occurs on the $\mathrm{C} 3$-carbon in 2-naphthyl. After that reaction goes through intermediate W6 (-46.97 kcal mol $\left.{ }^{-1}\right)$ and hydrogen-loss transition state TS6-P6 (-18.11 kcal $\mathrm{mol}^{-1}$ ) and finds its end in the product $\mathrm{P} 6+\mathrm{H}$ located at $-24.26 \mathrm{kcal} \mathrm{mol}^{-1}$. 


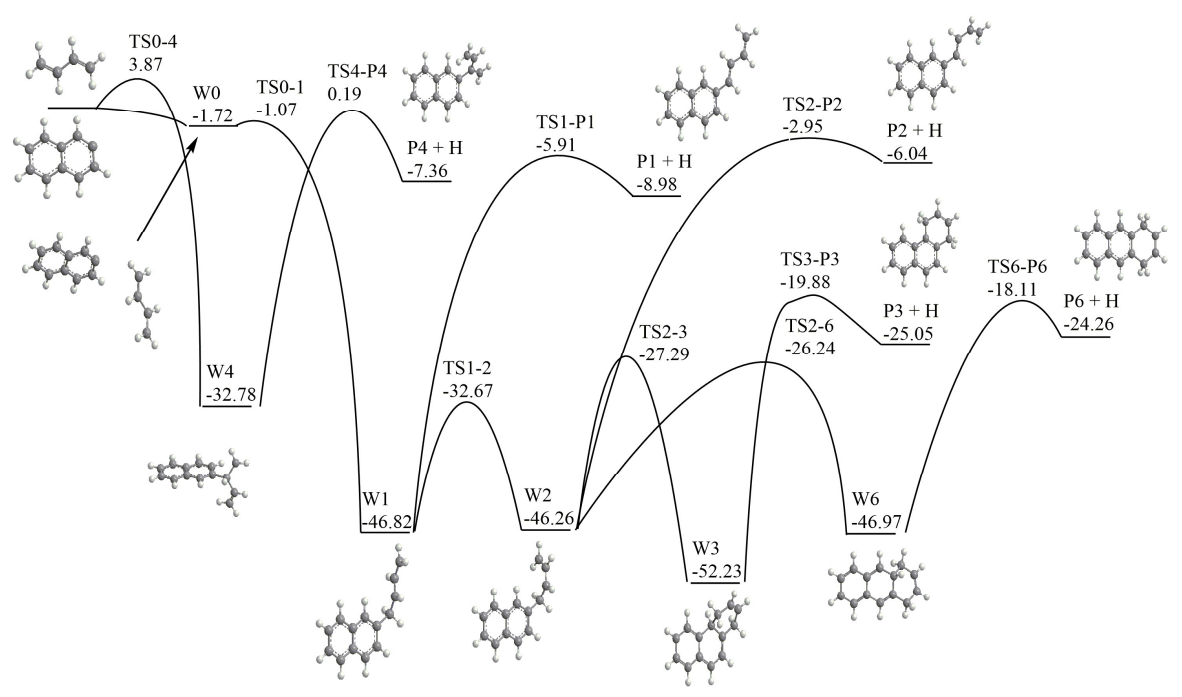

Fig. 1. Potential energy diagram for the reaction of 2-naphthyl with 1,3-butadiene; the energies are given in $\mathrm{kcal} \mathrm{mol}^{-1}$ relative to the separated reactants.

RRKM-Master Equation calculations were exploited to obtain reaction rate constants for five pressures indicated in Fig. 2 in the range of temperature from 300 to $2500 \mathrm{~K}$. The calculated temperature-dependent reaction rate constants for pressures $0.01,0.1,1,10,100$ atm and the high pressure limit are presented in Figure 2. The curves behaviour below 700 $\mathrm{K}$ is similar and coincides with the HP limit. Some small decrease could be seen for all finite pressures at higher temperatures and after that the rate constants merge in one curve again.

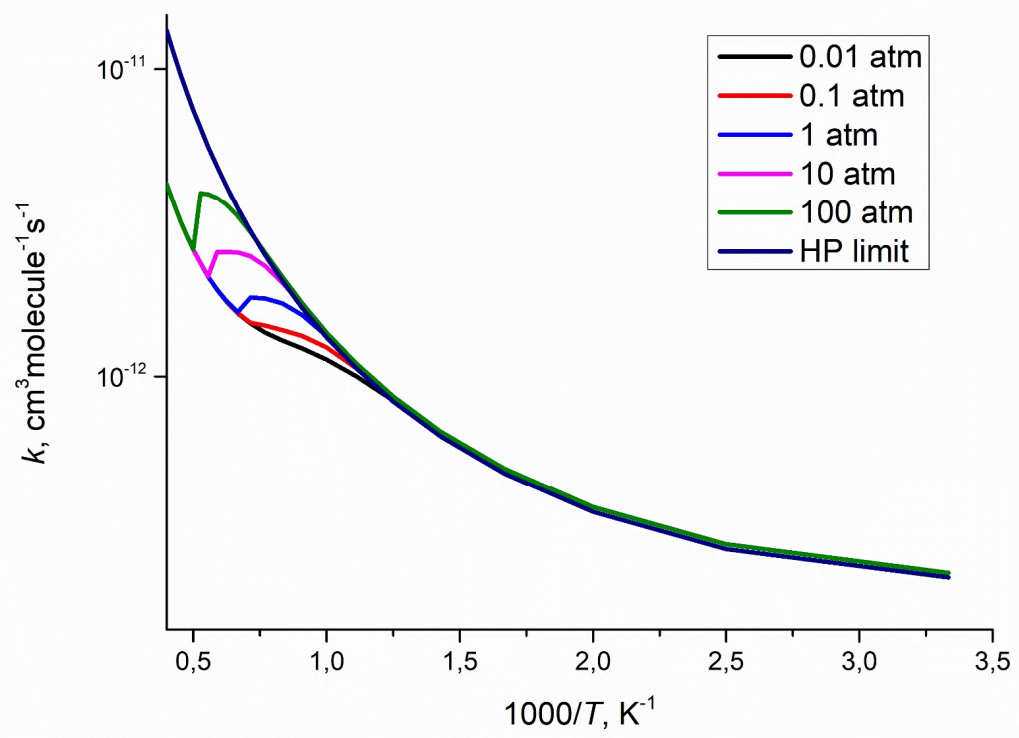

Fig. 2. Reaction rate constants for all investigated pressures and at the high pressure limit. 
The analysis of the branching ratios allows us to conclude that the channels leading P1 and $\mathrm{P} 2$ plus $\mathrm{H}$ would be the main channels in the reaction at high temperatures, whereas the three-ring products dihydrophananthrene P3 and dihydroanthracene P6 show significant yields in the 1200-2000 $\mathrm{K}$ range. The reaction shows low pressure dependence and the product branching ratios for P1-P6 are presented in Table 1. For the temperatures below $1200 \mathrm{~K}, \mathrm{~W} 1$ and W2 complexes get collisionally stabilized and the resulting contributions are presented in Table 2 .

Table 1. Branching ratios of the reaction of 2-naphthyl with 1,3-butadiene for pressure $1 \mathrm{~atm}$.

\begin{tabular}{|c|c|c|c|c|c|}
\hline $\mathbf{T}, \mathbf{K}$ & $\mathbf{P 1}$ & $\mathbf{P 2}$ & $\mathbf{P 3}$ & $\mathbf{P 4}$ & $\mathbf{P 6}$ \\
\hline 1200 & $3,08 \%$ & $1,44 \%$ & $4,13 \%$ & $0,02 \%$ & $2,93 \%$ \\
\hline 1300 & $28,89 \%$ & $14,39 \%$ & $32,61 \%$ & $0,27 \%$ & $22,87 \%$ \\
\hline 1400 & $35,98 \%$ & $19,47 \%$ & $25,38 \%$ & $0,62 \%$ & $18,56 \%$ \\
\hline 1500 & $41,30 \%$ & $24,01 \%$ & $19,28 \%$ & $0,81 \%$ & $14,60 \%$ \\
\hline 1600 & $45,09 \%$ & $27,91 \%$ & $14,61 \%$ & $1,00 \%$ & $11,38 \%$ \\
\hline 1700 & $47,64 \%$ & $31,16 \%$ & $11,14 \%$ & $1,17 \%$ & $8,89 \%$ \\
\hline 1800 & $49,26 \%$ & $33,82 \%$ & $8,60 \%$ & $1,33 \%$ & $6,99 \%$ \\
\hline 1900 & $50,25 \%$ & $36,00 \%$ & $6,72 \%$ & $1,47 \%$ & $5,56 \%$ \\
\hline 2000 & $50,79 \%$ & $37,79 \%$ & $5,34 \%$ & $1,60 \%$ & $4,48 \%$ \\
\hline 2100 & $51,05 \%$ & $39,27 \%$ & $4,30 \%$ & $1,72 \%$ & $3,65 \%$ \\
\hline 2200 & $51,13 \%$ & $40,51 \%$ & $3,51 \%$ & $1,84 \%$ & $3,01 \%$ \\
\hline 2300 & $51,10 \%$ & $41,54 \%$ & $2,91 \%$ & $1,94 \%$ & $2,51 \%$ \\
\hline 2400 & $51,01 \%$ & $42,40 \%$ & $2,44 \%$ & $2,04 \%$ & $2,12 \%$ \\
\hline 2500 & $50,88 \%$ & $43,12 \%$ & $2,06 \%$ & $2,13 \%$ & $1,80 \%$ \\
\hline
\end{tabular}

Table 2. Branching ratios of the $\mathrm{W} 1$ and $\mathrm{W} 2$ intermediates at the low temperatures for $1 \mathrm{~atm}$.

\begin{tabular}{|c|c|c|}
\hline $\mathbf{T}, \mathbf{K}$ & $\mathbf{W 1}$ & $\mathbf{W 2}$ \\
\hline 300 & $34,91 \%$ & $65,05 \%$ \\
\hline 400 & $33,36 \%$ & $66,49 \%$ \\
\hline 500 & $32,73 \%$ & $66,79 \%$ \\
\hline 600 & $29,82 \%$ & $69,08 \%$ \\
\hline 700 & & $98,21 \%$ \\
\hline 800 & & $97,72 \%$ \\
\hline 900 & & $97,40 \%$ \\
\hline 1000 & & $96,30 \%$ \\
\hline 1100 & & $93,76 \%$ \\
\hline 1200 & & $88,25 \%$ \\
\hline
\end{tabular}




\section{Conclusion}

The mechanism of the 2-naphthyl $+1,3$-butadiene reaction has been proposed in this study. The potential energy diagram and reaction rate constants with branching ratios were obtained in a broad set of pressures and temperatures. This study is important to complement the assumption PAH generation is not only a high-temperature phenomenon. $\mathrm{P} 1$ and $\mathrm{P} 2$ with hydrogen are concluded to be the main products at high temperatures but the three-ring products dihydrophenanthrene and dihidroanthracene can also be readily formed at certain conditions. The calculated reaction rate constants show low pressure dependence at pressures above $0.01 \mathrm{~atm}$.

The work was supported by the Ministry of Education and Science of the Russian Federation under the Grant No. 14.Y26.31.0020 to Samara University.

\section{References}

1. B.S. Haynes, H.G. Wagner, Prog. Energy Combust. Sci. 7 (1981).

2. M. Frenklach, Phys. Chem. Chem. Phys. 4 (2002).

3. H. Richter, J.B. Howard, Prog. Energy Combust. Sci. 26 (2000).

4. M. Frenklach, E. D. Feigelson, Astrophys. J. 341 (1989).

5. M. Frenklach, H. Wang, Symp. (Int.) Combust. 23 (1991).

6. I. Cherchneff, J. R. Barker, A. G. G. M. Tielens, Astrophys. J. 401 (1992).

7. B. Shukla, M. Koshi, Combust. Flame 158 (2011).

8. B. Shukla, K. Tsuchiya, M. Koshi M. 2011. J. Phys.Chem. A 115 (2011).

9. A. M. Mebel, V. V. Kislov, R. I. Kaiser. 2008 J. Am. Chem. Soc. 130 (2008).

10. A. M. Thomas, M. Lucas, T. Yang, R. I. Kaiser, L. Fuentes, D. Belisario-Lara, A. M. Mebel, ChemPhysChem 18, 15 (2017).

11. M.J. Frisch et al. GAUSSIAN 09, Revision A.1 (Gaussian, Inc., Wallingford, CT, 2009).

12. MOLPRO, version 2010.1, a package of ab initio programs, H.J.Werner and others, see http://www.molpro.net. 